\title{
Chemoprevention against colon cancer by dietary intake of sulforaphane
}

\section{Hideo Suzuki ${ }^{1}$, Michihiro Mutoh ${ }^{2}$, Toshiro Kamoshida ${ }^{3}$, Nobushige Kakinoki ${ }^{3}$, Shigemasa Yoshida ${ }^{4}$, Tsugio Ebihara ${ }^{5}$, Mitsuaki Hirose ${ }^{6}$, Ichinosuke Hyodo ${ }^{7}$, and Akinori Yanaka ${ }^{8}$}

${ }^{1}$ Department of Gastrointestinal Endoscopy, Faculty of Medicine, University of Tsukuba, Japan; ${ }^{2}$ Division of Prevention, National Cancer Center, Japan; ${ }^{3}$ Department of Gastroenterology, Hitachi General Hospital, Japan; ${ }^{4}$ Department of Gastroenterology, National Hospital Organization, Mito Medical Center, Japan; ${ }^{5}$ Deartment of Gastroenterology, Ryugasaki Saiseikai Hospital, Japan; ${ }^{6}$ Department of Gastroenterology, Kasumigaura Regional Medical Education Center, University of Tsukuba Hospital, Japan; ${ }^{7}$ Department of Gastroenterology, University of Tsukuba, Japan; ${ }^{8}$ Hitachi Medical Education and Research Center, Tsukuba University Hospital, Faculty of Medicine, University of Tsukuba, Japan.

Corresponding Author: Akinori Yanaka, MD and Ph.D., Hitachi Medical Education and Research Center, Tsukuba University Hospital, Faculty of Medicine, University of Tsukuba, Hitachi, Ibaraki 317-0077, Japan.

Submission Date: April $4^{\text {th }}, 2019$. Acceptance Date: June $28^{\text {th }}, 2019$. Publication Date: June $30^{\text {th }}, 2019$.

Citation: Suzuki H, Mutoh M, Kamoshida T, Kakinoki N, Yoshida S, Ebihara T, Hirose M, et al.: Chemoprevention Against Colon Cancer by Dietary Intake of Sulforaphane. Functional Foods in Health and Disease 2019, 9(6):392-411. DOI: https://doi.org/10.31989/ffhd.v9i6.607

\begin{abstract}
Background: Sulforaphane (SFN) is a phytochemical compound which belongs to isothiocyanates family found in abundance in broccoli sprouts. SFN induces a variety of antioxidant enzymes via NF-E2-related factor 2-Kelch-like ECH-associated protein 1mediated pathway, thereby protecting cells from injury induced by various kinds of oxidative stresses. We have previously shown that SFN protects gastric mucosa from oxidative injury induced by $H$. pylori infection. SFN also down-regulates histone deacetylase (HDAC) activity, thereby inducing apoptosis and inhibiting proliferation of tumor cells in variety of tissues. On the other hand, the incidence of colon cancer has increased in Japan.
\end{abstract}

Aim: Since numerous epidemiological studies have shown that colon cancer is inversely associated with intake of anti-oxidant vegetables, this study examines whether daily intake of SFN prevents colon tumorigenesis in mice and human subjects. 


\section{Methods}

\section{Effects of SFN on Colonic tumorigenesis in Mice Treated with Chemical Carcinogen:} Effects of SFN on colonic tumorigenesis were examined in the ICR male mice, pretreated with a chemical carcinogen, azoxymethane (AOM) $(15 \mathrm{mg} / \mathrm{kg})$. The mice treated with AOM for 3 or 6 times were fed for 8 or 24 weeks with or without sulforaphane glucosinolates (SGS: 2,200 $\mathrm{ppm} / \mathrm{kg} /$ day), which is a precursor of SFN. Effects of SGS treatment on formation of the microscopic aberrant crypt foci (ACF) and the macroscopic tumors in colonic mucosa were evaluated.

2. Effects of SFN on formation of Colonic ACF in patients with colonic adenoma: Effects of intake of raw broccoli sprouts (BS), $50 \mathrm{~g}$ /day containing $220 \mathrm{mg}$ SGS every other day, for 6 months on changes in the number of ACF in rectal mucosa was examined by colonoscopy in patients with colonic adenoma.

3. Effects of SFN on intestinal microbiota in human subjects: Effects of dietary intake of raw BS, $20 \mathrm{~g} /$ day, which contains $88 \mathrm{mg}$ SGS every other day, for 2 weeks on intestinal microbiota in healthy volunteers was assessed by measuring composition of stool bacteria, using a method of terminal restriction fragment length polymorphism flora analysis. In human studies, alfalfa sprouts, which contains no SFN, was used as a placebo control.

Results: 1. Daily administration of SGS suppressed formation of microscopic ACF and macroscopic colonic tumors in the AOM-pretreated mice in vivo. 2. Intake of BS for 6 months tended to decrease the number of colonic ACF in patients with colonic adenoma. 3. Intake of BS for 2 weeks increased percentages of Bifidobacterium and Clostridium XVIa, which has been shown to enhance protection of colonic mucosa by increasing butyrate production in colonic lumen.

Conclusions: 1. Daily intake of SFN affords chemoprotection against colonic tumors in the mice treated with a chemical carcinogen. 2 . The present study further suggests that, in addition to previously reported mechanisms, changes in the intestinal microbiota by SFN intake may also play a role in chemoprevention against colon cancer. Further studies are required to prove this possibility.

Keywords: sulforaphane, colon cancer, chemoprevention, aberrant crypt foci, intestinal microbiota

\section{INTRODUCTION}

Recent studies have demonstrated that colon cancer has been gradually increasing in Japan [1]. According to the data from National Cancer Center in Japan, colon cancer is the second largest cause of cancer death in males and the third in females in Japan [1]. A number of epidemiological studies have suggested that colon cancer is strongly associated with dietary habits $[1,2,3,4,5,6,7]$. For example, red meats and alcohols increase risk of colon cancer [3, $4,5]$, while fish, fruits, vegetables, and coffees reduce cancer risk [6, 7, 8]. Among various fruits and vegetables, cruciferous vegetables have been shown to possess strong preventive effects against various types of cancers [9], since cruciferous vegetables contain high amount of isothiocyanates, which show strong anti-oxidant activity, via NF-E2-related factor 2 (Nrf2)- 
Kelch-like ECH-associated protein 1 (Keap1) systems [10, 11, 12]. Recent studies have shown that sulforaphane, one of the compounds in isothiocyanate family, is especially rich in broccoli sprouts [13]. Sulforaphane (SFN) has been shown to induce various kinds of antioxidant enzymes via the nrf2-keap1 systems, thereby strengthening cytoprotective activity during exposure to oxidative stress [14]. Since many types of carcinogens continue to load chronic oxidative stress to cells, the intake of SFN on the regular basis may contribute to chemoprevention against cancers. In fact, several animal studies have shown that SFN prevents experimentally induced various types of cancers $[15,16]$. Our previous data obtained from mice experiments have shown that SFN mitigates oxidative injuries in gastrointestinal tract induced either by H.pylori infection or non-steroidal anti-inflammatory drugs (NSAIDs) treatment $[17,18]$. We have also demonstrated that these protective effects of SFN are induced at least in part by nrf2-dependent induction of anti-oxidant enzymes [17, 18]. Recent studies have shown that, in addition to the nrf2-mediated enhancement of anti-oxidant activity, SFN has other mechanisms which also contributes to chemoprotection against cancers. For example, SFN inhibits histone deacetylase (HDAC) activity of many types of tumor cells, which inhibits cell cycles and induces apoptosis in tumor cells [19, 20,21]. These mechanisms are almost identical with that of NSAIDs, which also contributes to cancer chemoprevention [22]. Furthermore, SFN has strong anti-bactericidal activity against gastric H.pylori [23]. Our previous studies have shown that SFN suppresses proliferation of gastric H.pylori, a wellknown gastric carcinogen $[17,24]$. SFN also inhibits growth of anaerobic bacterial in small intestinal mucosa, which causes injuries in small intestinal mucosa [18]. Recent studies by others have shown that SFN normalizes intestinal flora in the mice which has been suffering from chemically induced bladder cancer [25]. Based on these backgrounds, SFN may contribute to chemoprevention of colon cancer by normalizing intestinal microflora. The present study was conducted to determine whether SFN prevents colon cancers in mice and humans and to address the mechanisms by which SFN affords colon cancer chemoprotection.

\section{METHODS}

\section{Animal Study: Effect of Dietary SGS on AOM-Induced Colon Tumorigenesis in Mice}

In the animal study, we examined whether oral intake of SFN affect colon tumorigenesis in chemically-induced colon tumor model in mice. In this study, azoxymethane (AOM), a wellknown chemical carcinogen was administrated to ICR male mice at 6 weeks old age. This treatment was known to induce various types of colon tumors, such as microscopic aberrant crypt foci (ACF), and macroscopic tumors [26, 27] (Fig 1).

After feeding for 8 or 24 weeks, the mice were sacrificed under anesthesia by urethane. Colonic tissues were extracted and were fixed with $10 \%$ paraformaldehyde for 24 hours. The whole part of colonic mucosa in each mouse was examined by a stereoscopic microscopy. The number of tumors in the colonic mucosa in each mouse was counted. Subsequently, the colonic mucosae were stained with $0.2 \%$ methylene blue, and the number of aberrant crypt foci (ACF) in the whole colonic mucosa in each mouse was counted under a light microscopy. All animal experiments were performed according to the guidelines of the National Research Council Guide for the Care and Use of Laboratory Animals and approved by the Review Board of Tsukuba University. 


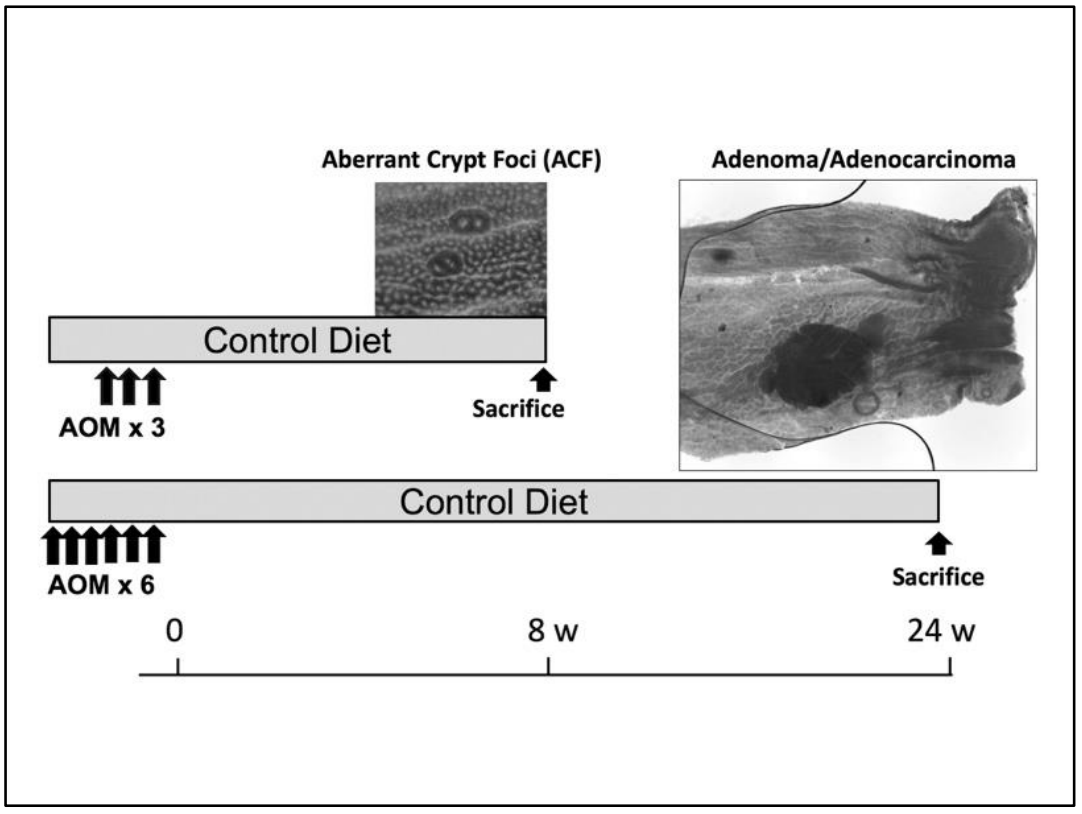

Figure 1. Experimental Protocol for Animal Study on the Effect of azoxymethane (AOM)treatment on Colonic Tumorigenesis in Mice. ICR male mice injected intracutaneously 3 or 6 times with $15 \mathrm{mg} / \mathrm{kg}$ azoxymethane, were fed with ordinary diet for 8 or 16 weeks, respectively.

The mice were divided into the following two groups. First group: The mice were injected percutaneously with AOM, $15 \mathrm{mg} / \mathrm{kg}$, once a week for 3 times. Then, the mice were fed with either the ordinary diet or the diet containing sulforaphane glucosinolates (SGS), 2,200 ppm/kg/day, for 8 weeks. SGS is a precursor of sulforaphane, which is not biologically active. However, once SGS was administrated into gastrointestinal lumen, SGS was gradually transformed to biological active SFN, through digestion by intestinal bacterial myrosinase activity. The second group: the mice were injected percutaneously with AOM, $15 \mathrm{mg} / \mathrm{kg}$, once a week for 6 times. Then the mice were fed with either the ordinary diet or the diet containing with SGS, 2,200 $\mathrm{ppm} / \mathrm{kg} / \mathrm{day}$, for 24 weeks (Fig 2).

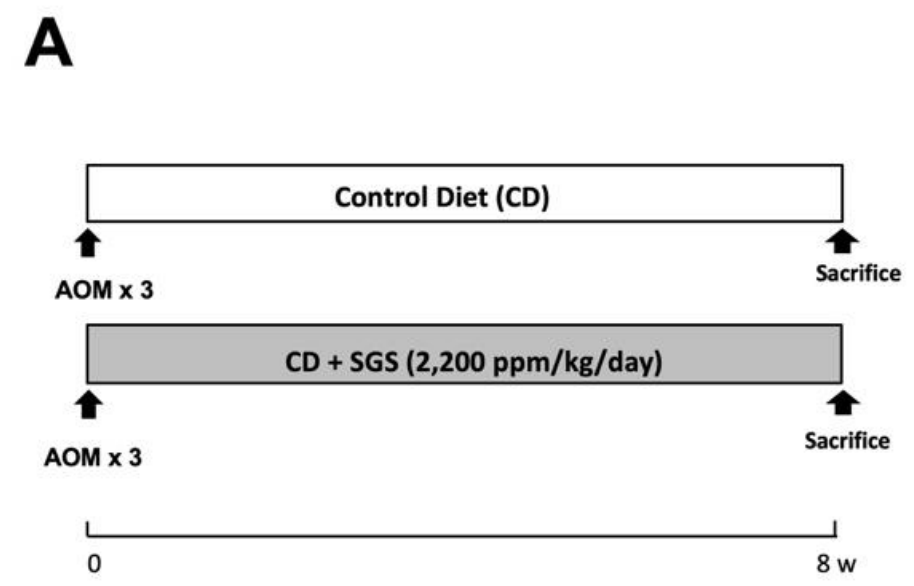




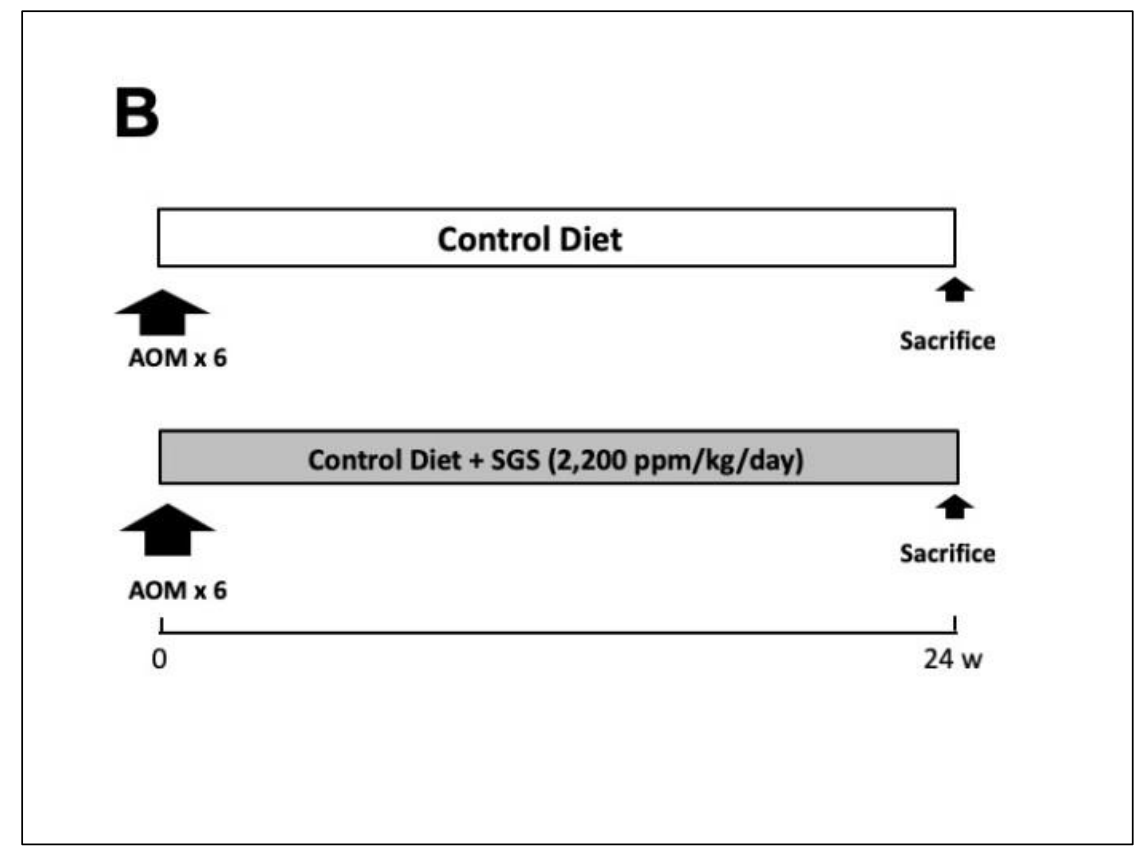

Figure 2. Experimental Protocol for Animal Study on the Effect of Dietary SGS on AOMinduced Colonic Tumorigenesis in Mice. ICR male mice at 6 weeks of age were pretreated with $15 \mathrm{mg} / \mathrm{kg}$ AOM for 6 times. Subsequently, the mice were fed with mice ordinary diet in the absence or the presence of 2,200 ppm sulforaphane glucosinolates (SGS) for 8 (A) or 24 weeks (B). All the mice were sacrificed, and the number of ACF and macroscopic tumors in colonic mucosa were counted.

\section{Human Study 1: Effect of BS Intervention on ACF in Rectal Mucosa}

This study was conducted to determine whether daily intake of SGS-rich broccoli sprouts (BS) would decrease the number of ACF, which is a surrogate marker for colon tumorigenesis. In order to evaluate whether daily intake of BS inhibits colon tumorigenesis in humans, an exploratory study by a single arm of BS intervention trial was designed. This study was approved by the ethical committee of each hospital participated in this study, and was registered with the University Hospital Medical Information Network in Japan (U-Min: Registration Number: UMIN000012022). The trial was registered as "Effect of Broccoli Sprouts Intervention on Chemoprevention against Colon Cancer". This study was conducted by 6 members of Tsukuba Cancer Clinical Trial Group (TCTG).

Twenty-three subjects, 15 males and 8 females, were recruited from patients with colonic adenoma, which was diagnosed by colonoscopy. The mean age of the subjects was 62.7, ranging from 49 to 76 years. All subjects agreed to participate in this study and signed a written informed consent form. The following exclusion criteria were applied: subjects suffering from inflammatory bowel diseases, subjects using NSAIDs, aspirin, or warfarin on a regular basis, and subjects with a broccoli allergy. A past or present history of GI disorders or disorders affecting other organs, such as liver, kidney, and endocrine organs, was also included as an exclusion criterion. No subjects were excluded from this study, based on these criteria. All the subjects were confirmed to have colon polyps, which had been pathologically diagnosed adenoma. The initial colonoscopy was performed before the BS intervention. After finishing a routine observation of total colon, all the polyps larger than $5 \mathrm{~mm}$ in diameter was 
endoscopically removed. Subsequently, rectal mucosa was stained with $0.2 \%$ methylene blue. The number of ACF in rectal mucosa was counted using a magnifying colonoscopy. The whole processes of ACF counting were recorded by a blue-ray recorder and saved as video and image files. The detail of these procedures were originally described by Takayama et al [28, 29].

\section{BS intervention: BS intervention was started within 1 week after the initial colonoscopy}

In this study, all the BS were cultured and harvested at Murakami Farm Inc., which contains $4.4 \mathrm{mg} / \mathrm{g}$ of SGS. The fresh raw BS was delivered to the participants' home or office twice a week during the treatment period. Subjects were requested to refrain from consuming broccoli sprouts personally purchased from supermarkets during the 6 month intervention period. During the intervention period, subjects were requested to eat $50 \mathrm{~g}$ of raw BS, which contains $220 \mathrm{mg}$ SGS, every other day for 6 months. In our previous study, we confirmed that oral intake of SGS, $30 \mathrm{mg} /$ day, up-regulates anti-oxidant enzyme activities, such as glutathione Stransferase (GST) and $\mathrm{NAD}(\mathrm{P}) \mathrm{H}$ : quinone oxidoreductase 1 (NQO1) in human healthy volunteers (30). Only one patient dropped out from the study because of abdominal pain, which began just a few days before the intervention. The second colonoscopy was conducted within 1 week after finishing the 6 month intervention with BS. The ACF counting was conducted by the same procedure as the first colonoscopy. The data obtained from one subjects were excluded from the following analysis, since most of the endoscopic images of rectal mucosa were not durable for precise counting of ACF, due to large amounts of mucus, which covered over the rectal mucosa (Fig 3). All the recorded image files from the remaining 21 subjects were blinded. The number of ACF was counted by two specialists for gastrointestinal endoscopy, who had not been aware of the condition of each data. All the images of ACF was classified into the 4 different groups; Hyperplastic ACF; Dysplastic ACF; Non Hyperplastic, Non-dysplastic ACF; Unclassified ACF, which has been described in detail elsewhere [28, 29].

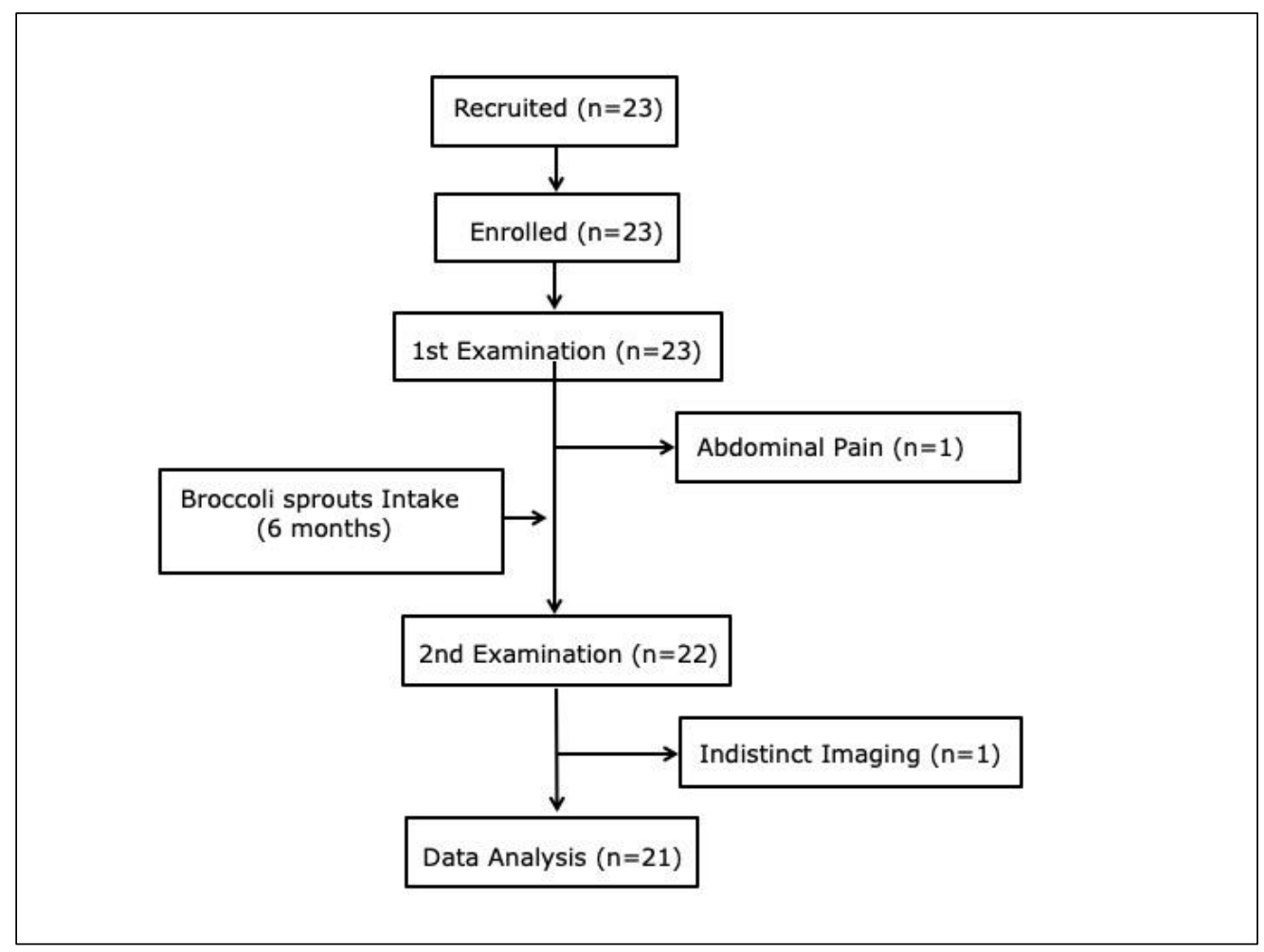

Figure 3. Protocol for Clinical Trial on the Effect of Broccoli Sprouts Intervention on Colonic 
ACF in patients with Colonic Adenoma. After the initial total colonoscopy by ordinary procedures, rectal mucosa in each participant were stained with $0.15 \%$ methylene blue. and, the number of ACF was counted using magnifying colonoscopy. Subsequently, all the subjects were fed with $50 \mathrm{~g}$ BS, which contains $128 \mathrm{mg}$ SGS, every other day for 6 months. Then the colonoscopy was performed and the number of ACF was counted in the same procedures as the first examination.

Human Study 2: Effects of BS or Alfalfa Sprouts (AS) Intervention on Intestinal Microbiota In order to evaluate whether daily intake of BS alters intestinal microbiota in healthy human volunteers, a placebo-controlled semi-open label intervention trial was designed. This study was approved by the ethical committee of the Hitachi General Hospital (Approval Number, 2017-85) and was registered with the University Hospital Medical Information Network in Japan (U-Min: Registration Number: UMIN-000032565). The trial is registered as "Effects of Dietary Intake of Broccoli Sprouts on Intestinal Microbiota and Defecation in Human Subjects -2nd Trial”.

Participants Recruitment: Twenty-four female healthy subjects with mild constipation were recruited from employees of the Hitachi General Hospital. All subjects agreed to participate in this study and signed a written informed consent form.

Protocol for Sprouts Intervention: In this study, alfalfa sprouts (AS) were used as placebo because AS contains virtually no SGS. BS contains $4.4 \mathrm{mg} / \mathrm{g}$ of SGS. According to the data from the manufacture, and the Standard Tables of Food Composition in Japan (http://www.mext.go.jp/a_menu/syokuhinseibun/1365297.htm), there was no significant difference between $\mathrm{BS}$ and $\mathrm{AS}$ in the composition of almost all other nutrients, minerals, vitamins, and dietary fibers. BS and the AS were commercially available. However, in this study, all the sprouts were cultured and harvested at Murakami Farm Co. Ltd, and the fresh raw sprouts were delivered to the participants' home or office twice a week during the treatment period. The gross appearance of AS resembles that of BS, although the taste of BS is rather spicy than that of BS. Subjects were not informed whether they were assigned to either the BS or the AS group. To exclude the potential effects of some foods and drugs on the intestinal microbiota, subjects were requested to refrain from consuming cruciferous vegetables, fermented foods, laxatives, probiotics, and antibiotics, throughout the entire trial period. This study was conducted using a cross-over method. All the subjects were allocated into 2 groups. Allocation was conducted to minimize differences in age, body weight, and body mass index between the two groups (Table 1). Half of the 24 recruited participants were initially treated with BS $20 \mathrm{~g} /$ day for 2 weeks. After the ensuing wash-out period for 2 weeks, the subjects were treated with AS $20 \mathrm{~g} /$ day for 2 weeks. The other halves were treated with the same amount of AS initially, followed by 2 weeks wash-out period, and the ensuing 2 week period of the BS intake (Fig 4). During the intervention period, subjects were requested to eat either $20 \mathrm{~g}$ of raw BS or AS every day for 4 weeks. After the 2-week pre-trial period, stool samples were collected on 4 occasions (i.e. immediately before and after the first and second intervention).

Sample Analysis: Stool samples were collected to evaluate intestinal microflora. Composition of the intestinal microflora in each stool samples was evaluated using a terminal restriction fragment length polymorphism (T-RFLP) flora analysis, Nagashima's method [31], which 
revealed the percentage of Bifidobacterium, Lactobacillus's, Bacteroides, Prevotella, Clostridium [cluster IV, IX, XI, XIVa, XVIII], and others. Stool sample analyses were performed at Techno Suruga Lab, Shizuoka, Japan.

Table 1. Subjects Profile in the Studies on the effects of BS/AS on Intestinal Microbiota.

\begin{tabular}{ccc}
\hline & BS $\rightarrow$ AS & AS $\rightarrow$ BS \\
\hline \# of Subjects & 12 & 12 \\
Gender (Male:Female) & $0: 12$ & $0: 12$ \\
Age (years) & $36.8 \pm 8.54$ & $37.8 \pm 8.29$ \\
Height (cm) & $162 \pm 2.25$ & $157 \pm 8.15^{a}$ \\
Weight (kg) & $56.9 \pm 8.35$ & $56.5 \pm 8.99$ \\
Body Mass Index & $21.5 \pm 3.02$ & $22.9 \pm 2.84$ \\
\hline
\end{tabular}

Values are expressed as Mean $\pm S D$. a, $P<0.05$ : Significant Difference from the corresponding value in the AS group.

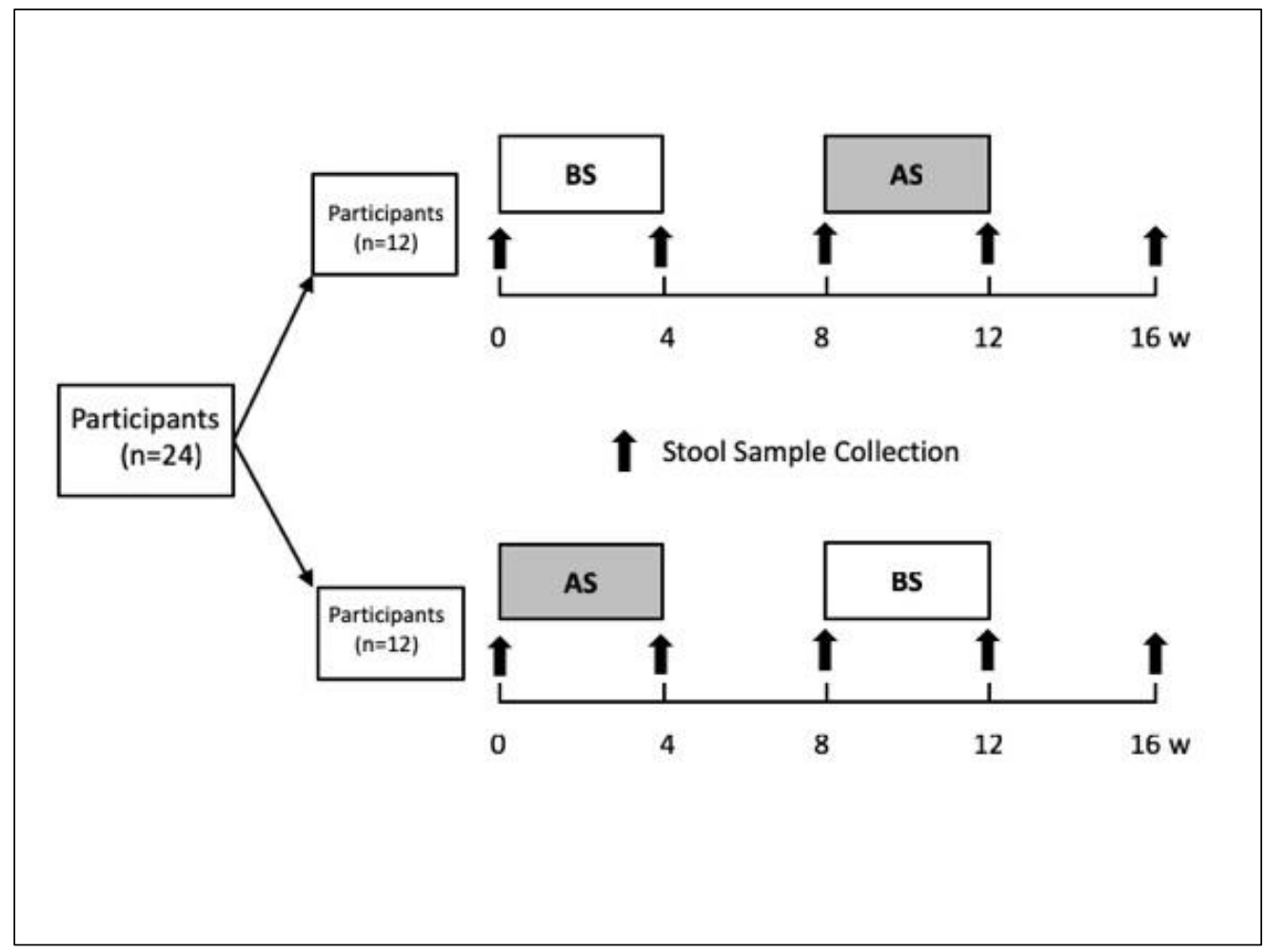

Figure 4. Protocol for Clinical trial on the Effects of Dietary intervention with Broccoli/Alfalfa Sprouts on Human Intestinal Microbiota. This intervention trial was conducted by a crossover method. All the subjects were assigned to take BS or AS $20 \mathrm{~g} / \mathrm{day}$ for 2 weeks. Intestinal microbiota was assessed by TRFLP analysis, using stool samples collected before and after taking BS or AS. 


\section{Data Analysis}

The student's t-test was used for analysis of continuous data with a normal distribution. Nonparametric tests were used for continuous data that did not show a normal distribution and with discrete data. For non-parametrical analysis, the Wilcoxon signed-rank test and the MannWhitney $\mathrm{U}$ test were applied for comparison of unpaired and paired data, respectively. $P$ values less than 0.05 were considered to be statistically significant. Pearson's chi-square test was applied to analysis of the changes in the intestinal microbiota after sprouts intake.

\section{RESULTS}

\section{Animal Study: Effect of Dietary SGS on AOM-Induced Colon Tumorigenesis in Mice}

In the AOM-untreated mice, feeding with or without SGS for 8 or 24 weeks caused neither $\mathrm{ACF}$ nor tumors in colonic mucosa. In the mice pretreated 3 times with AOM, feeding with ordinary diet for 8 weeks caused colonic ACF. In the mice pretreated 6 times with AOM, feeding with ordinary diet for 24 weeks caused various types of macroscopic colonic tumors, such as hyperplastic polyp, tubular adenoma, and tubular adenocarcinoma (Fig 5). Feeding the AOM-pretreated mice with SGS for 8 or 24 weeks significantly reduced the number of colonic $\mathrm{ACF}$, and decreased the number of macroscopic tumors in colonic mucosa (Fig 6).

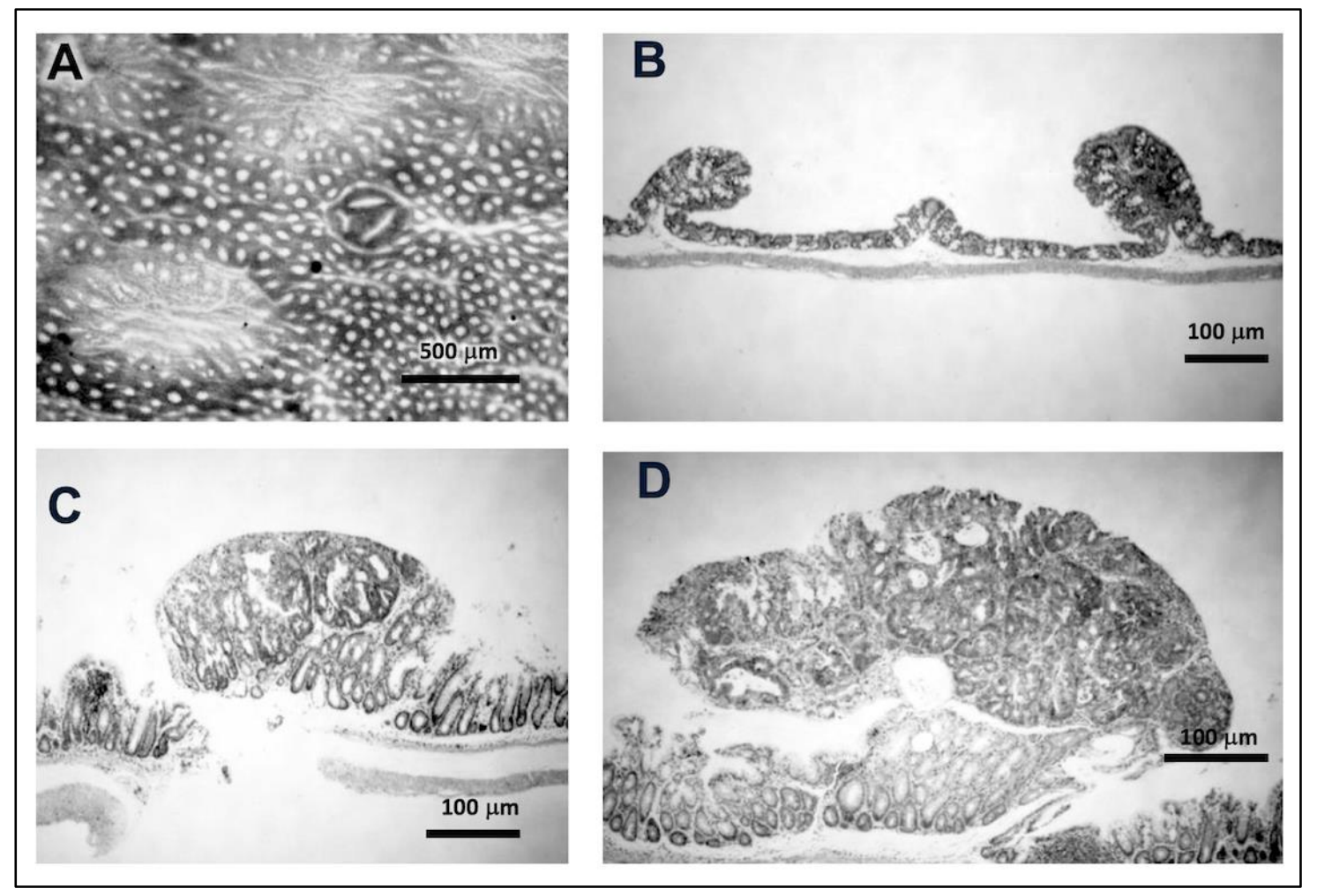

Figure 5. Effects of AOM-treatment on Colon tumorigenesis in ICR Mice. AOM-pretreated mice for 3 times developed aberrant crypt foci (ACF) in colonic mucosa 8 weeks later, while the mice pretreated with AOM 6 times developed various types of macroscopic colon tumors 24 weeks later. A; ACF, B; hyperplastic polyp, C; tubular adenoma, D; tubular adenocarcinoma. 


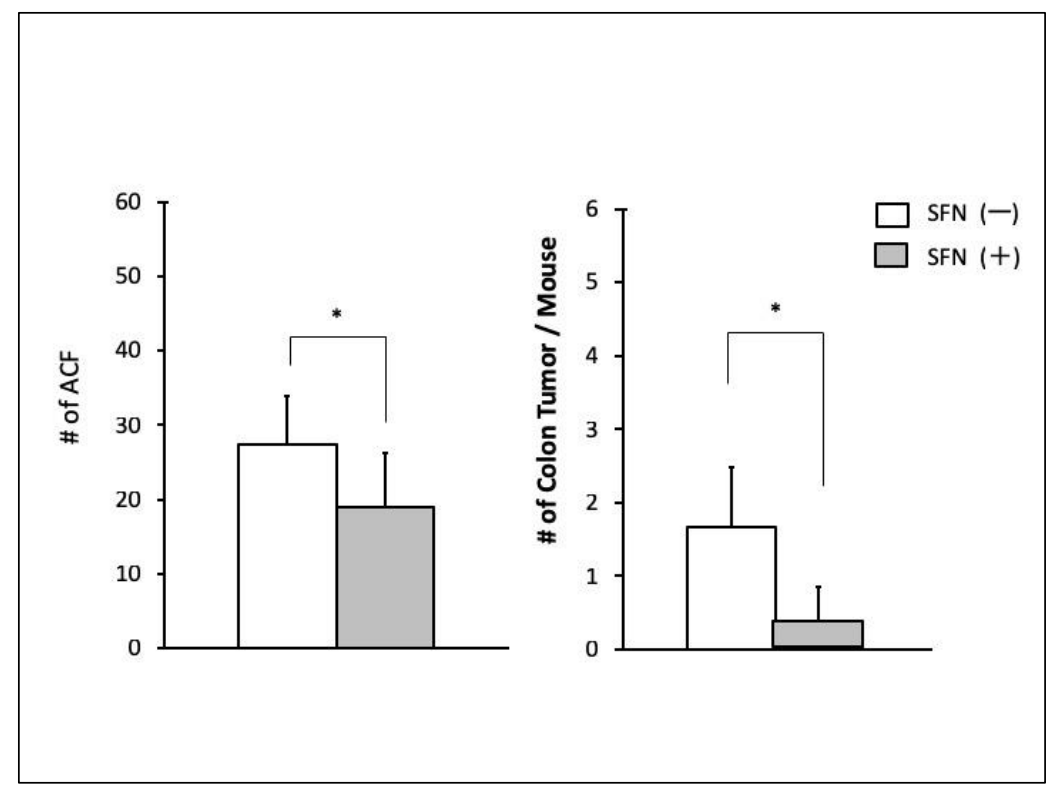

Figure 6. Effects of Dietary SGS on Colon Tumorigenesis in Mice. The number of colonic $\mathrm{ACF}$ at 8 weeks after the AOM pretreatment was significantly smaller in the SGS-treated than the SGS-untreated mice. The number of colonic macroscopic tumors at 24 weeks after the AOM pretreatment was significantly smaller in the SGS-treated than the SGS-untreated mice.

\section{Human Study 1: Effect of BS Intervention on Aberrant Crypt Foci in Rectal Mucosa}

All the participants completed 6 month trial with BS, without showing any adverse reactions. There was no statistically significant change in the number of rectal ACF after the 6 month treatment with BS. (Fig 7, Table 2).

Table 2. Changes in Various Types of Aberrant Crypt Foci after Dietary Intervention with Broccoli Sprouts in Human Subjects. The mean number of each type of ACF decreased after intake of BS, although the decreases in the ACF number were not statistically significant.

Type of ACF

\# of ACF $\quad($ Mean $\pm S D, n=21)$

\section{Before BS Intake $\quad$ After BS Intake}

Total ACF

$14.1 \pm 10.1$

$12.6 \pm 8.95$

Non-Hyperplastic

Non-Dysplastic ACF

$7.90 \pm 8.12$

$7.19 \pm 7.24$

Hyperplastic ACF

$1.86 \pm 2.08$

$1.62 \pm 2.73$

Dysplastic ACF

$1.29 \pm 1.68$

$1.09 \pm 2.43$

Unclassified ACF

$3.05 \pm 3.17$

$2.67 \pm 2.13$ 


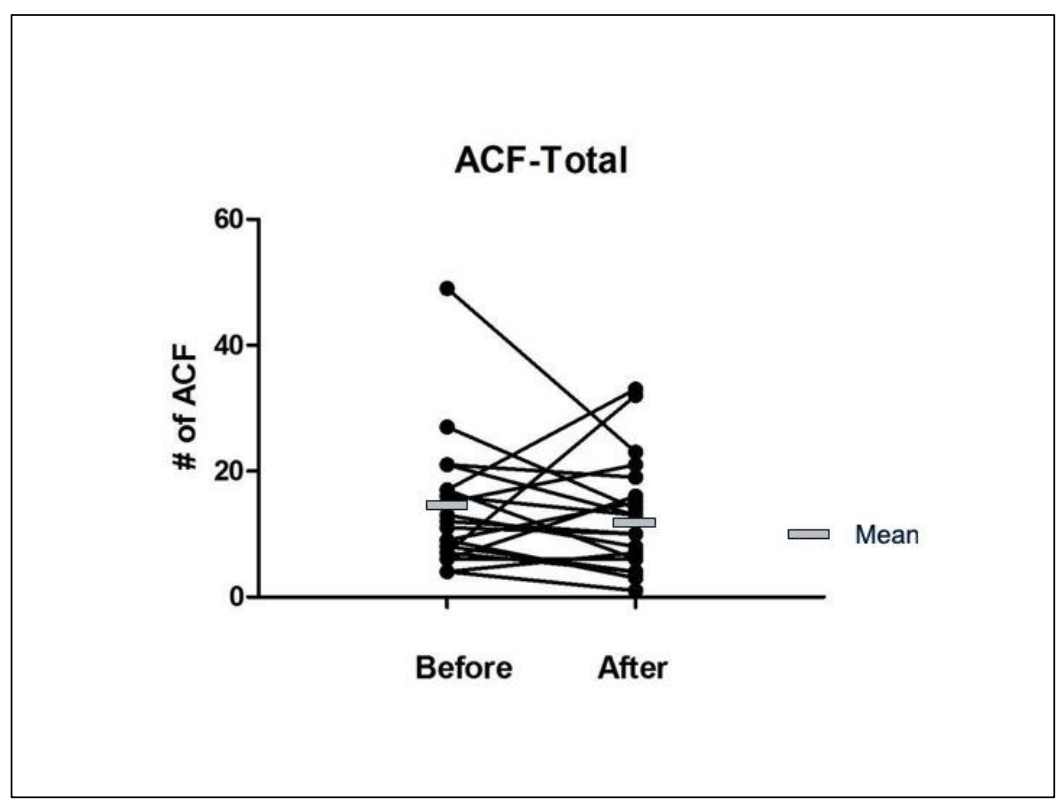

Figure 7. Changes in Aberrant Crypt Foci in Rectal Mucosa after 6 Months Dietary Intervention with Broccoli Sprouts in Human Subjects.

Changes in the total number of ACF after the BS intervention. [B] Changes in the number of various types of $\mathrm{ACF}$ after the intervention. The mean number of each type of ACF decreased after intake of BS, although the changes were not statistically significant.

\section{Human Study 2: Effects of BS or AS Intervention on Intestinal Microbiota}

T-RFLP analysis of the stool samples from the participants, showed Bifidobacterium, Lactobacillus, Bacteroides, Prevotella, Clostridium (IV, IX, XI, XIVa, XVIII), and others as intestinal microbiota. There were no significant changes in the mean values of the prevalence of each type of intestinal microbiota after the BS or the AS treatment. However, BS treatment significantly increased prevalence of Clostridium XIVa in 16 out of 23 subjects (69.5\%), while the AS treatment increased Bifidobacterium only in 7 out of the 23 subjects $(39.1 \%)(\mathrm{P}=0.038$ : BS vs AS). Furthermore, BS treatment tended to increase prevalence of Bifidobacterium in 15 out of 23 subjects $(68.2 \%)$, while the AS treatment increased Bifidobacterium only in 9 out of the 23 subjects $42.9 \%)(\mathrm{P}=0.095$ : BS vs AS) (Table 3$)$.

Table 3. Changes in Pattern of Intestinal Microbiota after Dietary Intervention with Broccoli or Alfalfa Sprouts in Human Subjects.

\begin{tabular}{lccc}
\hline A & \multicolumn{3}{c}{ Broccoli Sprouts Intervention $(\mathrm{n}=23)$} \\
Intestinal Microbiota & $1 \mathrm{st}$ & 2nd & 3rd \\
\hline Bifidobacterium & $12.1 \pm 8.7$ & $11.7 \pm 9.6$ & $12.3 \pm 9.7$ \\
Lactobacillales & $4.3 \pm 5.4$ & $3.3 \pm 3.6$ & $4.2 \pm 5.0$ \\
Bacteroides & $46.1 \pm 12.5$ & $45.3 \pm 13.4$ & $46.5 \pm 15.0$
\end{tabular}




\begin{tabular}{lccc} 
Prevotella & $4.9 \pm 11.6$ & $5.9 \pm 13.7$ & $4.6 \pm 11.7$ \\
Clostridium IV & $6.3 \pm 4.4$ & $6.1 \pm 4.8$ & $4.5 \pm 3.4$ \\
Clostridium XIVa & $12.7 \pm 5.4$ & $13.1 \pm 5.3$ & $12.7 \pm 5.1$ \\
Clostridium IX & $5.0 \pm 6.5$ & $5.9 \pm 8.0$ & $7.2 \pm 8.7$ \\
Clostridium XI & $0.8 \pm 1.7$ & $0.2 \pm 0.4$ & $0.4 \pm 0.6$ \\
Clostridium XVIII & $1.5 \pm 1.4$ & $1.5 \pm 1.3$ & $1.4 \pm 1.0$ \\
Others & $6.1 \pm 4.5$ & $7.0 \pm 5.2$ & $6.3 \pm 4.6$ \\
\hline
\end{tabular}

B

Alfalfa Sprouts Intervention $(n=23)$

\begin{tabular}{lccc}
\multicolumn{1}{c}{ Intestinal Microbiota } & $1 \mathrm{st}$ & 2nd & 3rd \\
\hline Bifidobacterium & $12.7 \pm 9.2$ & $12.0 \pm 8.2$ & $12.0 \pm 8.3$ \\
Lactobacillales & $3.5 \pm 3.2$ & $3.4 \pm 3.0$ & $3.9 \pm 4.7$ \\
Bacteroides & $45.8 \pm 13.7$ & $47.5 \pm 11.8$ & $48.3 \pm 11.1$ \\
Prevotella & $5.6 \pm 12.7$ & $3.3 \pm 9.8$ & $3.2 \pm 8.4$ \\
Clostridium IV & $5.0 \pm 2.8$ & $5.3 \pm 3.4$ & $5.4 \pm 4.5$ \\
Clostridium XIVa & $12.1 \pm 7.1$ & $11.7 \pm 7.0$ & $12.9 \pm 5.4$ \\
Clostridium IX & $5.1 \pm 7.5$ & $5.8 \pm 6.6$ & $6.1 \pm 6.6$ \\
Clostridium XI & $0.6 \pm 0.8$ & $1.1 \pm 3.3$ & $0.5 \pm 0.6$ \\
Clostridium XVIII & $1.4 \pm 1.3$ & $1.1 \pm 1.0$ & $1.3 \pm 1.2$ \\
Others & $5.6 \pm 4.1$ & $7.4 \pm 7.1$ & $6.5 \pm 4.2$ \\
\hline C & & & $\boldsymbol{P}$ value \\
\hline Increase & Decrease &
\end{tabular}

\section{Bifidobacterium}

Broccoli Sprouts $(n=22)$

Alfalfa Sprouts $\quad(n=21)$

15

7

9

12

0.095

\section{Clostridium XIVa}

Broccoli Sprouts $(n=23)$

Alfalfa Sprouts $\quad(n=23)$

16

9

7

14

0.038

$[A, B]$ There were no changes in the mean values of percentages of each bacteria, after taking BS or AS. [C] There was a significant difference in the individual change pattern in Bifidobacterium, and Clostridium XVIa, between the BS and the AS treatment. Compared with AS treatment, BS intake significantly increased Clostridium XVIa, and tended to increase Bifidobacterium. 


\section{DISCUSSION}

The data on mice experiments in the present study demonstrates that oral intake of SGS significantly inhibits both ACF formation and tumorigenesis in colonic mucosa in AOMpretreated mice. We have previously shown that oral administration of similar amounts of SGS up-regulates antioxidant enzymes, such as glutathione S-transferase (GST) and NAD(P)H: quinone oxidoreductase 1 (NQO1) and hem oxygenase 1 (HO-1), and inhibits oxidative injury in gastrointestinal mucosa in mice $[17,18]$. We have also demonstrated in our previous study that oral intake of 30 to $60 \mathrm{mg}$ SGS enhances serum levels of GST and NQO-1 activities in human healthy subjects [30]. Therefore, it seems reasonable to assume that SGS mitigates oxidative stress induced by the AOM treatment, thereby suppressing ACF formation and tumorigenesis in colon.

Another mechanism of the inhibition of colon tumorigenesis by SFN appears to be related closely with its inhibitory effect on HDAC activity. The cell cycle has been suggested to be regulated by a balance between histone acetylation and deacetylation, which are induced by histone acetyl transferase (HAT) and histone deacetylase (HDAC), respectively [32]. Since HDAC plays an important role in the cell proliferation [32], it seems likely that up-regulation of HDAC activity frequently observed in many types of cancer cells is closely associated with the enhancement of tumor cell growth [33]. A number of recent studies have demonstrated that SFN inhibits HDAC activity [19, 20, 21], thereby inhibiting cell proliferation and inducing apoptosis of tumor cells [21]. In the present study, we have also observed that SGS treatment in a few cases of mice inhibited HDAC activity and induced apoptosis in the mice treated with AOM + dextran sodium sulfate (unpublished observation). Thus, chemoprevention by SGS may also be induced as a result of HDAC inhibition. We need to evaluate further the effect of SGS on HDAC activity in mice colon in the present experimental systems. Based on the results from the mice experiments, we hypothesized that SFN also protects human colonic mucosa from tumorigenesis, since our results in the clinical trial with BS have shown that the mean values of the ACF number in all types of ACF slightly decreased after the BS intake, even though the observed changes were not statistically significant. There are several possible explanations for the reasons that we failed to observe a significant effect of SFN in human colonic ACF in the present study. First, in some cases large amounts of mucus, which covered the colonic mucosa, may have disturbed the precise counting of the ACF number. Second, the present study was conducted in 4 different hospitals using difference types of colonoscope by different physicians. Although multicenter study is able to collect large numbers of data in a 
short period of time without bias, there is a drawback in a larger variation in the data than those obtained from a single hospital. Third, it is also conceivable that counting error of ACF by an each endoscopist may be too large to demonstrate small changes in the ACF number induced by BS intake. Furthermore, it may be also possible that the inhibitory effects of BS on the ACF was masked by a natural increase in the ACF number during the 6 month period. In order to solve these problems, we need to compare the results with the data obtained from placebo trial in the future studies.

The data on the human study 2 also suggest that, in addition to the mechanisms mentioned above, SFN may contribute to chemoprevention against colon cancer by suppressing mucosal invasion of harmful bacteria in the colonic mucosa, since we found that BS intake increases Bifidobacterium and Clostridium XIVa, both of which have been shown to enhance butyrate production in colonic lumen [34, 35, 36, 37, 38, 39]. A number of previous studies have shown that various types of short chain fatty acid, such as butyrate, modulate various colonic functions and protects colonic epithelial cells from carcinogenesis [40, 41, 42, 43, 44]. Thus, it seems conceivable that increased production of butyrate by Bifidobacerium and Clostridium XIVa after intake of SFN may also afford chemoprevention against colon cancer. We have previously discovered that treatment with BS or SGS suppresses colonization of H.pylori in gastric mucosa [17] and inhibits growth of anaerobic bacteria in the small intestinal mucosa [18]. Brussels sprouts, which also contains large amounts of SFN, have been reported to modulate composition of intestinal microbiota [34]. Thus, it seems reasonable to assume that SFN normalizes intestinal microbiota, which may also contribute to chemoprotection against colon cancer. In order to examine whether the inhibitory effect of SFN in colon tumorigenesis is causally related with the increase in butyrate producing bacteria, another placebo-controlled colonic adenoma-intervention trials should be conducted in the future studies, in which we need to examine the effects of SFN not only on the ACF number but also on the gut microbiota and butyrate concentrations in stool and mucosal samples using the same human patients. We also need to examine the effects of SFN on some key genes and miRNA in colonic tissues, which are closely associated with the mutagenesis of colonic mucosa.

Strategies for colon cancer chemoprevention trials have been conducted mainly using drugs such as aspirin or NSAIDs. However, a number of clinical trials have demonstrated that long-term use of aspirin or COX-2 inhibitors increases serious adverse reactions such as gastrointestinal bleeding or cardiovascular events, which prevents long term administration to these agents to health human subjects. In contrast, dietary approach by food components have 
lower risk of serious adverse reaction, thereby being a potentially better option for long-term administration than drugs, even though the preventive effect may be milder than drugs. Additionally, given the high prevalence of colon cancer and its partial age-association, dietary anti-oxidant intervention approach may be envisaged also as an amenable "antiaging" protective intervention, as proposed by other investigators [45].

\section{CONCLUSIONS}

The present study showed that daily intake of SFN affords chemoprotection against colonic tumors in the mice treated with a chemical carcinogen. The present study suggests that, in addition to previously reported mechanisms, such as up-regulation of the antioxidant enzymes and the down-regulation of HDAC activity, changes in the intestinal microbiota by SFN intake may also play a role in chemoprevention against colon cancer. Further studies are required to prove this possibility (Fig 8).

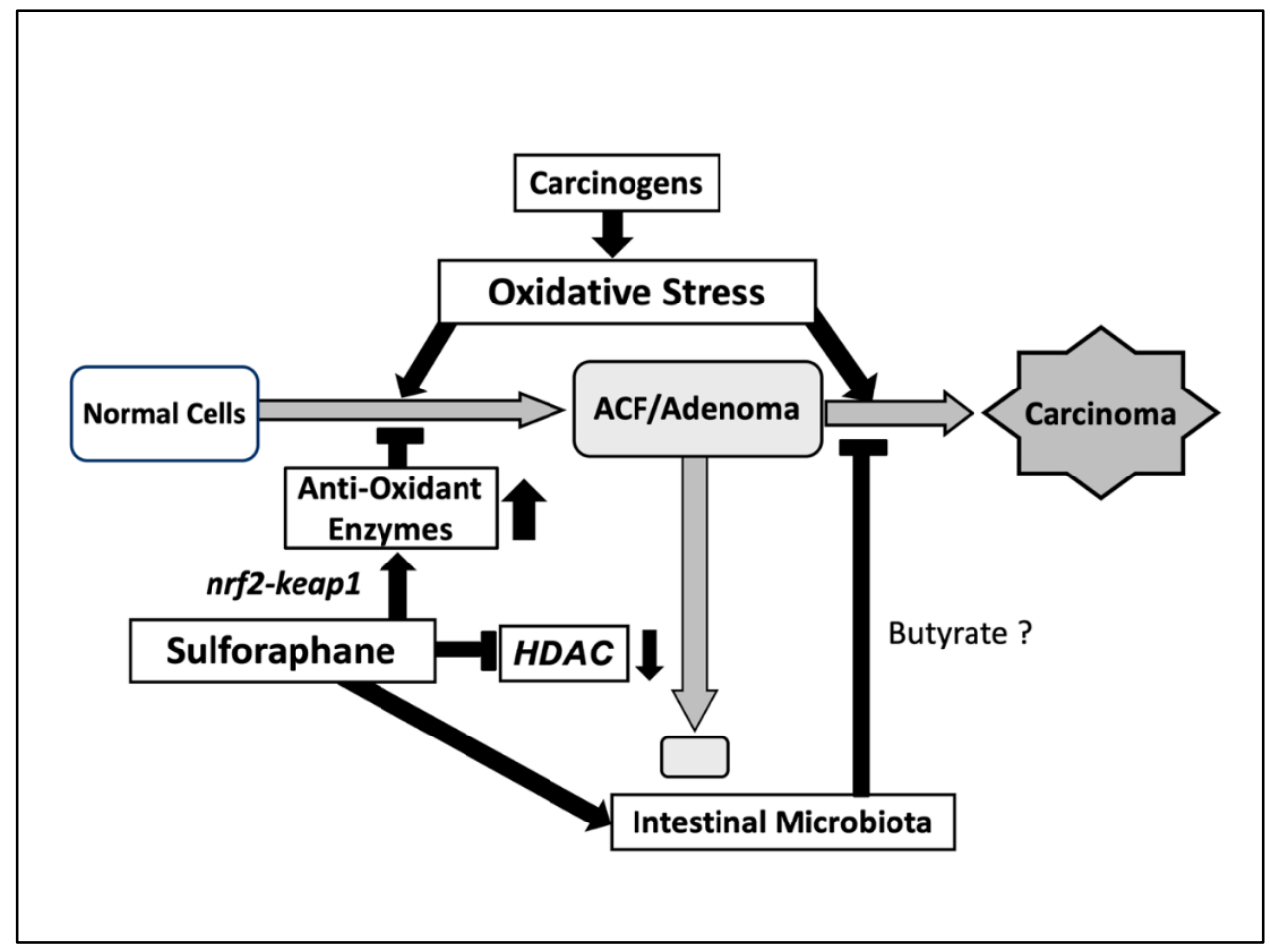

Figure 8. Putative Mechanisms by Which Sulforaphane Afford Colon Cancer Chemoprevention. (1) Sulforaphane prevents oxidative injury in colonic mucosa via upregulating nrf2-keap1 system. (2) Sulforaphane inhibits proliferation, and induces apoptosis of tumor cells, via inhibition of histone-deacetylase activity. (3) Sulforaphane may prevents tumorigenesis by normalizing intestinal microbiota.

List of Abbreviations: ACF, aberrant crypto foci; AOM, azoxymethane; AS, alfalfa sprouts; BS, broccoli sprouts; H. pylori, Helicobacter pylori; HDAC, histone deacetylase; Keap-1, Kelch-like ECH-associated protein 1; Nrf2, NF-E2-related factor 2; NSAIDs, non-steroidal 
anti-inflammatory drugs; SFN, sulforaphane; SGS, sulforaphane glucosinolates; T-RFLP, terminal restriction fragment length polymorphism.

Competing Interests: Akinori Yanaka is an endowed chair of Hitachi Medical Education and Research Center in Tsukuba University Hospital, funded by Hitachi Co. Ltd. Human studies were financially supported in part by Murakami Farm Inc.

Authors' Contributions: Akinori Yanaka, and Hideo Suzuki equally contributed to whole part of the study. Michihiro Mutoh contributed to planning animal study design. Toshiro Kamoshida contributed to pathological assessment of mice tissues. Shigemasa Yoshida, Tsugio Ebihara, Mitsuaki Hirose, and Ichinosuke Hyodo contributed to human study 1. Nobushige Kakinoki contributed to human study 2 .

Acknowledgements and Funding: The human study 1 was supported in part by members of Tsukuba Cancer Clinical Trial Group. The authors would gratefully thank Drs. Kazuto Ikezawa, and Takunori Ueno, for independent review of the ACF image files in human study 1. Animal experiments were supported by Japan Society for the Promotion of Science, KAKENHI Grant Number 24501000. Human studies were financially supported in part by Murakami Farm Inc.

\section{REFERENCES:}

1. Watanabe T, Muro K, Ajioka Y, Hashiguchi Y, Ito Y, Saito Y, Hamaguchi T, et al.: Japanese Society for Cancer of the Colon and Rectum (JSCCR) guidelines 2016 for the treatment of colorectal cancer. Int J Clin Oncol 2018, 23(1):1-34.

2. Pan JH, Abernathy B, Kim YJ, Lee JH, Kim JH, Shin EC, Kim JK: Cruciferous vegetables and colorectal cancer prevention through microRNA regulation: A review. Crit Rev Food Sci Nutr 2018, 58(12):2026-2038.

3. Thanikachalam K, Khan G: Colorectal Cancer and Nutrition. Nutrients 2019, 11(1):pii:E164.

4. Pham NM, Mizoue T, Tanaka K, Tsuji I, Tamakoshi A, Matsuo K, Wakai K, et al.: Meat consumption and colorectal cancer risk: an evaluation based on a systematic review of epidemiologic evidence among the Japanese population. Jpn J Clin Oncol 2014, 44(7):641-50.

5. Fedirko V, Tramacere I, Bagnardi V, Rota M, Scotti L, Islami F, Negri E, et al.: Alcohol drinking and colorectal cancer risk: an overall and dose-response metaanalysis of published studies. Ann Oncol 2011, 22(9):1958-1972. 
6. Kashino I, Mizoue T, Tanaka K, Tsuji I, Tamakoshi A, Matsuo K, Wakai K, et al.: Vegetable consumption and colorectal cancer risk: an evaluation based on a systematic review and meta-analysis among the Japanese population. Jpn J Clin Oncol 2015, 45(10):973-9.

7. Pham NM, Mizoue T, Tanaka K, Tsuji I, Tamakoshi A, Matsuo K, Wakai K, et al.: Fish consumption and colorectal cancer risk: an evaluation based on a systematic review of epidemiologic evidence among the Japanese population. Jpn J Clin Oncol 2013, 43(9):935-41.

8. Akter S, Kashino I, Mizoue T, Matsuo K, Ito H, Wakai K, Nagata C, et al.: Coffee drinking and colorectal cancer risk: an evaluation based on a systematic review and meta-analysis among the Japanese population. Jpn J Clin Oncol 2016, 46(8):781-7.

9. Murillo G, Mehta RG: Cruciferous vegetables and cancer prevention. Nutr Cancer 2001, 41(1-2):17-28.

10. Zhang Y, Tang L: Discovery and development of sulforaphane as a cancer chemopreventive phytochemical. Acta Pharmacol Sin 2007, 28(9):1343-1354.

11. Yang L, Palliyaguru DL, Kensler TW: Frugal chemoprevention: targeting Nrf2 with foods rich in sulforaphane. Semin Oncol 2016, 43(1):146-153.

12. Munday R, Munday CM: Induction of phase II detoxification enzymes in rats by plant-derived isothiocyanates: comparison of allyl isothiocyanate with sulforaphane and related compounds. J Agric Food Chem 2004, 52(7):1867-1871.

13. Fahey JW, Zhang Y, Talalay P: Broccoli sprouts: an exceptionally rich source of inducers of enzymes that protect against chemical carcinogens. Proc Natl Acad Sci U S A 1997, 94(19):10367-72.

14. Chikara S, Nagaprashantha LD, Singhal J, Horne D, Awasthi S, Singhal SS: Oxidative stress and dietary phytochemicals: Role in cancer chemoprevention and treatment. Cancer Lett 2018, 413:122-134.

15. Gerhauser C: Epigenetic impact of dietary isothiocyanates in cancer chemoprevention. Curr Opin Clin Nutr Metab Care 2013, 16(4):405-10.

16. Gills JJ, Jeffery EH, Matusheski NV, Moon RC, Lantvit DD, Pezzuto JM: Sulforaphane prevents mouse skin tumorigenesis during the stage of promotion. Cancer Lett 2006, 236(1):72-9.

17. Yanaka A, Fahey JW, Fukumoto A, Nakayama M, Inoue S, Zhang S, Tauchi M, et al.: Dietary sulforaphane-rich broccoli sprouts reduce colonization and attenuate 
gastritis in Helicobacter pylori infected mice and humans. Cancer Prev Res (Phila) 2009, 2(4):353-360.

18. Yanaka A, Sato J, Ohmori S: Sulforaphane protects small intestinal mucosa from aspirin/ NSAID-induced injury by enhancing host defense systems against oxidative stress and by inhibiting mucosal invasion of anaerobic enterobacteria. Curr Pharm Des 2013, 19(1):157-162.

19. Tortorella SM, Royce SG, Licciardi PV, Karagiannis TC: Dietary sulforaphane in chemoprevention: The role of epigenetic regulation and HDAC inhibition. Antioxid Redox Signal 2015, 22(16):1382-424.

20. Ho E, Clarke JD, Dashwood RH: Dietary sulforaphane, a histone deacetylase inhibitor for cancer prevention. J Nutr 2009, 139(12):2393-6.

21. Myzak MC, Karplus PA, Chung FL, Dashwood RH: A novel mechanism of chemoprotection by sulforaphane: inhibition of histone deacetylase. Cancer Res 2004, 64(16):5767-74.

22. Antonakopoulos N, Karamanolis DG: The role of NSAIDs in colon cancer prevention.

23. Hepatogastroenterology 2007, 54(78):1694-700.

24. Fahey JW, Haristoy X, Dolan PM, Kensler TW, Scholtus I, Stephenson KK, Talalay P, et al.: Sulforaphane inhibits extracellular, intracellular, and antibiotic-resistant strains of Helicobacter pylori and prevents benzo[a]pyrene-induced stomach tumors. Proc Natl Acad Sci U S A 2002, 99(11):7610-5.

25. Yanaka A: Role of sulforaphane in protection of gastrointestinal tract against $\mathrm{H}$. pylori and NSAID-induced oxidative stress. Curr Pharm Des 2017, 23(27):40664075.

26. He C, Huang L, Lei P, Liu X, Li B, Shan Y: Sulforaphane normalizes intestinal flora and enhances gut barrier in mice with BBN-induced bladder cancer. Mol Nutr Food Res 2018, 62(24):e1800427.

27. Archer MC, Bruce WR, Chan CC, Corpet DE, Medline A, Roncucci L, Stamp D, et al.: Aberrant crypt foci and microadenoma as markers for colon cancer. Environ Health Perspect 1992, 98:195-7. 
28. Corpet DE, Pierre F: Point: From animal models to prevention of colon cancer. Systematic review of chemoprevention in min mice and choice of the model system. Cancer Epidemiol Biomarkers Prev 2003, 12(5):391-400.

29. Takayama T, Katsuki S, Takahashi Y, Ohi M, Nojiri S, Sakamaki S, Kato J, et al.: Aberrant crypt foci of the colon as precursors of adenomas and cancer. New Eng $\mathbf{J}$ Med 1998, 339(18):1277-1284.

30. Takahashi H, Takayama T, Yoneda K, Endo H, Iida H, Sugiyama M, Fujita K, et al.: Association of visceral fat accumulation and plasma adiponectin with rectal dysplastic aberrant crypt foci in a clinical population. Cancer Sci 2009, 100(1):29-32.

31. Ushida Y, Suganuma H, Yanaka A: Low-Dose of the Sulforaphane Precursor Glucoraphanin as a Dietary Supplement Induces Chemoprotective Enzymes in Humans. Food and Nutrition Sciences 2015, 6(17):1603-1612.

32. Nagashima K, Mochizuki J, Hisada T, Suzuki S, Shimomura K: Phylogenetic analysis of $16 \mathrm{~S}$ ribosomal RNA gene sequences from Human fecal microbiota and improved utility of terminal restriction fragment length polymorphism profiling. Biosci Microflora 2006, 25(3):99-107.

33. Wang C, Fu M, Mani S, Wadler S, Senderowicz AM, Pestell RG: Histone acetylation and the cell-cycle in cancer. Front Biosci 2001, 6:D610-29.

34. Tang Z, Ding S, Huang H, Luo P, Qing B, Zhang S, Tang R: HDAC1 triggers the proliferation and migration of breast cancer cells via upregulation of interleukin-8. Biol Chem 2017, 398(12):1347-1356.

35. Humblot C, Bruneau A, Sutren M, Lhoste EF, Dore J, Andrieux C, Rabot S: Brussels sprouts, inulin and fermented milk alter the faecal microbiota of human microbiotaassociated rats as shown by PCR-temporal temperature gradient gel electrophoresis using universal, Lactobacillus and Bifidobacterium 16S rRNA gene primers. Br J Nutr 2005, 93(5):677-84.

36. Rios-Covian D, Gueimonde M, Duncan SH, Flint HJ, de los Reyes-Gavilan CG: Enhanced butyrate formation by cross-feeding between Faecalibacterium prausnitzii and Bifidobacterium adolescentis. FEMS Microbiol Lett 2015, 362(21):pii:fnv176. 
37. Sugahara H, Odamaki T, Fukuda S, Kato T, Xiao JZ, Abe F, Kikuchi J, et al.: Probiotic Bifidobacterium longum alters gut luminal metabolism through modification of the gut microbial community. Sci Rep 2015, 5:13548.

38. Kanauchi O, Fujiyama Y, Mitsuyama K, Araki Y, Ishii T, Nakamura T, Hitomi Y, et al.: Increased growth of Bifidobacterium and Eubacterium by germinated barley foodstuff, accompanied by enhanced butyrate production in healthy volunteers. Int $\mathbf{J}$ Mol Med 1999, 3(2):175-184.

39. Moens F, De Vuyst L: Inulin-type fructan degradation capacity of Clostridium cluster IV and XIVa butyrate-producing colon bacteria and their associated metabolic outcomes. Benef Microbes 2017, 8(3):473-490.

40. Van den Abbeele P, Belzer C, Goossens M, Kleerebezem M, De Vos WM, Thas O, De Weirdt R, et al.: Butyrate-producing Clostridium cluster XIV a species specifically colonize mucins in an in vitro gut model. ISME J 2013, 7(5):949-61.

41. Borges-Canha M, Portela-Cidade JP, Dinis-Ribeiro M, Leite-Moreira AF, PimentelNunes P: Role of colonic microbiota in colorectal carcinogenesis: a systematic review. Rev Esp Enferm Dig 2015, 107(11):659-71.

42. Wang SY, Li JY, Xu JH, Xia ZS, Cheng D, Zhong W, Lai Y, et al.: Butyrate suppresses abnormal proliferation in colonic epithelial cells under diabetic state by targeting HMGB1. J Pharmacol Sci 2019, 139(4):266-274.

43. Inagaki A, Hayashi M, Andharia N, Matsuda H: Involvement of butyrate in electrogenic K+ secretion in rat rectal colon. Pflugers Arch 2019, 471(2):313-327.

44. Chen J, Vitetta L: Inflammation-Modulating Effect of Butyrate in the Prevention of Colon Cancer by Dietary Fiber. Clin Colorectal Cancer 2018, 17(3):e541-e544.

45. McNabney SM, Henagan TM: Short Chain Fatty Acids in the Colon and Peripheral Tissues: A Focus on Butyrate, Colon Cancer, Obesity and Insulin Resistance. Nutrients 2017, 9(12):pii:E1348.

46. Vaiserman AM, Koliada AK, Marotta F. Gut microbiota: A player in aging and a target for anti-aging intervention. Ageing Res Rev 2017, 35:36-45. 